\title{
G asto energético y composición corporal en mujeres con obesidad severa y mórbida sometidas a bypass gástrico
}

\author{
Fernando Carrasco ${ }^{1}$, Pamela Rojas ${ }^{1}$, Manuel Ruz ${ }^{1 a}$, \\ Anabella Rebolledo ${ }^{1 b}$, Juana Codoceo ${ }^{1 c}$, Jorge Inostrozalc, \\ Claudio Mizón ${ }^{1}$, Karin Papapietro², Attila Csendes ${ }^{2}$, \\ Jorge Rojas², Fernando Pizarro3c, Manuel 0 livares $^{3}$. \\ Energy expenditure and \\ body composition in severe and morbid \\ obese women after gastric bypass
}

Background: The effects of gastric bypass (GBP) on resting energy expenditure (REE) are not well known. Aim: To evaluate the changes in REE and its relationship with body composition in severe and morbid obese women before and six and twelve months after GBP. Patients and methods: Twenty three women aged $37 \pm 10$ years, with a body mass index of $44 \pm 4 \mathrm{~kg} / \mathrm{m}^{2}$, were evaluated before, six and twelve months after GBP. REE was measured in a Deltatrac indirect calorimeter and expressed as kcal/day. Fat mass (FM), and fat free mass (FFM) were determined by double beam X ray densitometry (DEXA). Results: Body weight reduction six and twelve months after GBP was $29.0 \pm 4.3$ and $35.8 \pm 6.9 \%$, respectively. The best predictor of weight reduction was initial weight $(\mathrm{p}<0.01)$. At six and twelve months, REE decreased by $291.7 \pm 260.0$ and $353.8 \pm 378.4 \mathrm{kcal} /$ day, respectively. In the same periods REE/kg body weight increased by 3.3 and $4.8 \mathrm{kcal} / \mathrm{kg}$ respectively, compared to baseline. REE/kg FFM was unchanged. Conclusions: GBP was associated with significant changes in body composition after six and twelve months. However, despite weight reduction, resting energy expenditure per fat free mass unit did not change significantly (Rev Méd Chile 2008; 136: 570-7).

(Key words: Energy metabolism; Gastric bypass; Obesity morbid)
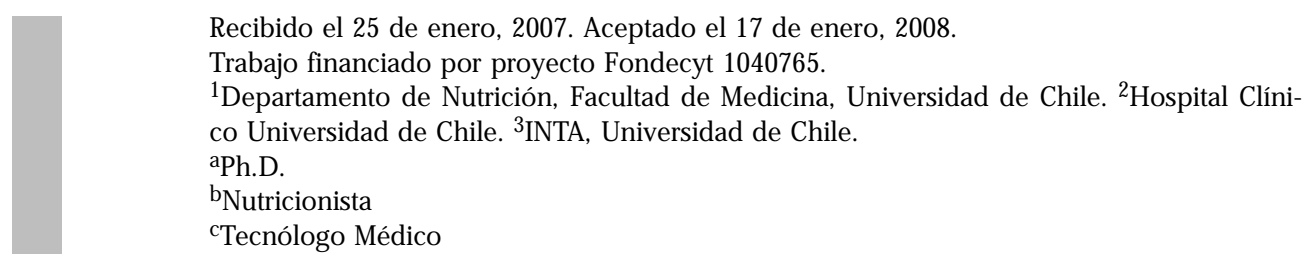

Correspondencia a: Dr. Fernando Carrasco. Independencia 1027. Santiago, Chile. Fono: 9786136 . Fax: 7378778.

E mail: fcarrasc@med.uchile.cl 
$\mathrm{E}^{\mathrm{1}}$ sobrepeso y la obesidad están considerados entre los principales problemas de salud pública, afectando a alrededor de 1,7 billones de individuos en todo el mundo ${ }^{1-3}$. Chile ha vivido un acelerado proceso de transición epidemiológica y nutricional, con un predominio de enfermedades crónicas no transmisibles ${ }^{4}$. La primera Encuesta Nacional de Salud corroboró la alta prevalencia de estas patologías en la población general mayor de 17 años: 38\% presenta sobrepeso, $22 \%$ obesidad y $1,3 \%$ obesidad mórbida; las dos últimas condiciones son más prevalentes en mujeres $^{5}$. Dado que la obesidad constituye un factor de riesgo para múltiples patologías, tales como diabetes mellitus tipo 2, dislipidemia, hipertensión arterial, apnea obstructiva del sueño, cardiopatías y trastornos psiquiátricos ${ }^{1,6,7}$, se emplean diversos tratamientos. Sin embargo, en pacientes con obesidad severa y mórbida el tratamiento médico multidisciplinario ha mostrado un reducido grado de eficacia ${ }^{2}$. En cambio, el tratamiento quirúrgico permite lograr una mejor y más sostenida reducción de peso en estos pacientes ${ }^{8,9}$, favoreciendo además una mejoría eficaz de las patologías asociadas a la obesidad ${ }^{10,11}$. Las técnicas más utilizadas en la actualidad son la banda gástrica ajustable y el bypass gástrico (BPG). El BPG es la técnica más común en Chile y en la mayoría de los países en el mundo, siendo considerado en la actualidad como el gold standard ${ }^{10-13}$.

El efecto del BPG sobre la reducción de peso es similar en los estudios realizados, pero sus efectos sobre el gasto energético de reposo (GER) son contradictorios. Algunos estudios muestran disminución del GER poscirugía ${ }^{14}$, que en algunos casos excede a la pérdida de masa corporal ${ }^{15,16}$; sin embargo, al analizar por subgrupos, en pacientes hipometabólicos el GER se tiende a normalizar y en normometabólicos se tiende a reducir, dentro de rangos normales ${ }^{17}$. Es importante establecer en este grupo de pacientes si se produce una disminución del GER (adaptación metabólica negativa), porque tendrán, al menos en teoría, un mayor riesgo de reganancia del peso a largo plazo. Además, resulta de interés evaluar la relación de estos cambios con las modificaciones de la composición corporal, porque existe escasa información al respecto ${ }^{18}$.

El objetivo del presente trabajo fue evaluar, en pacientes sometidos a bypass gástrico, los cambios en el gasto energético y composición corporal al sexto y duodécimo mes postoperatorio.

\section{PACIENTES Y MÉTODO}

Veintitrés mujeres con obesidad severa o mórbida, con edad de 36,4ะ9,8 años, y con indicación de BPG de acuerdo a los criterios de selección para tratamiento quirúrgico basados en el NIH Consensus Development Panel on Gastrointestinal Surgery for Severe Obesity ${ }^{12,19}$, ingresaron a un protocolo prospectivo en el cual se observaron las variaciones en el metabolismo energético en un seguimiento hasta el duodécimo mes post bypass gástrico. El estudio fue aprobado por el Comité de Ética de la Facultad de Medicina y del Hospital Clínico de la Universidad de Chile (HCUCH). Todas las pacientes firmaron un consentimiento informado.

Técnica quirúrgica. Todas las pacientes fueron operadas en el HCUCH, con una técnica descrita en detalle anteriormente ${ }^{10}$. Se realizó gastrectomía de 95\% (subtotal), dejando un reservorio gástrico vertical de $20 \mathrm{ml}$ de capacidad en la curvatura menor. La anastomosis gastroyeyunal se realizó con stapler circular № 25 (Tyco Healthcare). El largo del asa alimentaria en Y-de-Roux fue de $150 \mathrm{~cm}$.

En el preoperatorio (mes 0), al sexto (mes 6) y duodécimo mes (mes 12) postoperatorio se evaluaron: parámetros antropométricos, metabolismo y composición corporal, en dependencias del Departamento de Nutrición de la Facultad de Medicina.

Antropometría. Se midieron el peso corporal y la talla en una balanza digital Seca ${ }^{\circledR}$ (Vogel \& Halke $\mathrm{GmbH} \& \mathrm{Co}$, Alemania), con una precisión de \pm $100 \mathrm{~g}$; las pacientes fueron evaluadas descalzas y con ropa ligera. Con los datos obtenidos se calculó el índice de masa corporal $(\mathrm{IMC}=$ peso $(\mathrm{kg}) /$ talla $\left.(\mathrm{m})^{2}\right)$ ).

Gasto energético de reposo. Las determinaciones se realizaron después de un ayuno de $12 \mathrm{~h}$, período durante el cual tampoco podían fumar, tomar café, ni realizar actividad física. Las mediciones se realizaron alrededor de las 8:00 AM, en un ambiente termoestable (20-21ํㅡ), y después de 30 min de reposo. El GER se midió a través de 
calorimetría indirecta de circuito abierto ${ }^{20,21}$, con un monitor metabólico Deltatrac ${ }^{\circledR}$ (Datex Inst. Corp., Helsinki, Finlandia). Las determinaciones se efectuaron sólo después de que los sujetos alcanzaron un estado estable, con un coeficiente de variación intrasujeto de $\pm 5 \%$ a $10 \%$ en las mediciones de consumo de oxígeno $\left(\mathrm{VO}_{2}\right)$. Una vez lograda la estabilidad en el $\mathrm{VO}_{2}$, se registró el promedio de los últimos 5-10 min de evaluación. Con las mediciones de $\mathrm{VO}_{2}$ y producción de $\mathrm{CO}_{2}$ se calculó el GER con las fórmulas incorporadas en el monitor metabólico ${ }^{21}$.

Composición corporal. Los sujetos fueron ubicados en posición supina y se realizó absorciometría dual de rayos-X (DXA; Lunar DPX-L, Madison, WI; software 1.3), con una velocidad de barrido lenta. Con esta evaluación se determinó la masa grasa (MG) y la masa libre de grasa (MLG) en cuerpo entero.

Ingesta alimentaria. Se realizó una encuesta de registro de 24 h que incluyó dos días de la semana y un día del fin de semana. La información recolectada fue procesada mediante el programa de computación Food Processor II (Food Processor II. Nutrition Análisis System. Version 3.13. Salem: ESHA, 1989).

Estadística. Los resultados están expresados como promedio \pm desviación estándar. El test de ANOVA de muestras repetidas seguido por test de Bonferroni se aplicó para comparar las determinaciones pre y postoperatorias. La correlación entre varia- bles se evaluó con el coeficiente de Pearson. Se realizó regresión lineal simple y regresión lineal múltiple según método Stepwise para determinar los mejores predictores de reducción de peso y del GER. El análisis estadístico fue realizado con el programa SPSS 10.0 (SPSS Inc., Chicago, Illinois). Se aceptó como significativo un valor de $\mathrm{p}<0,05$.

\section{RESULTADOS}

Antropometría y composición corporal. En la Tabla 1 se presenta el peso, IMC y composición corporal de las voluntarias al momento previo de la cirugía, y a los 6 y 12 meses posteriores a la intervención. La reducción de peso fue de $29,0 \% \pm 4,3 \%$ al sexto mes post BPG y $35,8 \% \pm 6,9 \%$ al duodécimo mes, respecto del valor inicial. La reducción de peso correspondió a masa libre de grasa en $36 \%$ al mes 6 y $29 \%$ al mes 12. Todos los parámetros evaluados cambiaron en forma significativa al mes 6 y 12 con respecto al mes 0 . Además, el IMC, el porcentaje de MG y la masa grasa en kilos presentaron una disminución significativa entre el mes 6 y el mes 12 postoperatorio.

La reducción de peso al mes 6 (peso inicial peso mes 6) se correlacionó en forma positiva con los siguientes parámetros del mes 0: peso ( $\mathrm{r}=0,701$; $\mathrm{p} \varangle 0,001)$; MLG ( $\mathrm{r}=0,582 ; \mathrm{p}<0,01)$; $\mathrm{MG} \mathrm{kg}(\mathrm{r}=0,610$; $\mathrm{p} \varangle 0,01)$ y GER $(\mathrm{r}=0,557 ; \mathrm{p} \varangle 0,01)$. La reducción de peso no se correlacionó con edad, cuociente respiratorio, porcentaje de oxidación de lípidos ni con el porcentaje de MG. Al incorporar en un análisis de regresión múltiple 9 posibles variables de predicción

\section{Tabla 1. Cambios en la antropometría y composición corporal en 23 pacientes con seguimiento hasta el año postoperatorio}

\begin{tabular}{|lccc|}
\hline Parámetro & Mes 0 & Mes 6 & Mes 12 \\
\hline Peso $(\mathrm{kg})$ & $114,3 \pm 14,5^{\mathrm{a}}$ & $81,1 \pm 10,9^{\mathrm{b}}$ & $73,1 \pm 10,1^{\mathrm{c}}$ \\
IMC $\left(\mathrm{kg} / \mathrm{m}^{2}\right)$ & $44,5 \pm 3,7^{\mathrm{a}}$ & $31,6 \pm 3,4^{\mathrm{b}}$ & $28,5 \pm 3,5^{\mathrm{c}}$ \\
MG $(\%)$ & $47,5 \pm 4,8^{\mathrm{a}}$ & $40,6 \pm 6,0^{\mathrm{b}}$ & $33,2 \pm 7,6^{\mathrm{c}}$ \\
MG $(\mathrm{kg})$ & $54,8 \pm 11,3^{\mathrm{a}}$ & $33,4 \pm 9,0^{\mathrm{b}}$ & $24,9 \pm 8,4^{\mathrm{c}}$ \\
MLG $(\mathrm{kg})$ & $59,6 \pm 6,0^{\mathrm{a}}$ & $47,7 \pm 4,0^{\mathrm{b}}$ & $48,3 \pm 4,0^{\mathrm{b}}$ \\
N & 23 & 23 & 23 \\
\hline
\end{tabular}

IMC: índice de masa corporal; MG (\%): porcentaje de masa grasa; MG (kg): masa grasa en kilos; MLG (kg): masa libre de grasa en kilos. Supraíndice con letra distinta en la misma fila significa que las diferencias son estadísticamente significativas ( $\mathrm{p}<0,05$ ). 
de la reducción de peso, sólo fue significativo el peso inicial $\left(r=0,701 ; r^{2}=0,492 ; p<0,001\right)$.

La reducción de peso al mes 12 (peso inicial peso mes 12) se correlacionó con los siguientes parámetros iniciales: edad $(\mathrm{r}=0,423 ; \mathrm{p}<0,05)$; peso (0,722; $\mathrm{p}<0,001)$; MG kg ( $\mathrm{r}=0,672 ; \mathrm{p}<0,001)$ y GER $(r=0,547, p<0,01)$. Al mes 12 , el peso inicial también fue la variable que mejor predijo la reducción de peso $\left(r=0,722 ; r^{2}=0,521 ; p<0,001\right)$.

Metabolismo energético e ingesta alimentaria. La Tabla 2 muestra las variaciones del metabolismo energético durante el estudio. El GER disminuyó en promedio $291,7 \pm 260,0 \mathrm{kcal} /$ día al mes 6 y en $353,8 \pm 378,4 \mathrm{kcal} /$ día al mes 12 respecto del mes 0 ( $\mathrm{p}<0,001)$.

El GER/kg de peso corporal aumentó al mes 6 en $3,3 \mathrm{kcal} / \mathrm{kg} /$ día y en $4,8 \mathrm{kcal} / \mathrm{kg} /$ día al mes 12 (p $<0,001)$ respecto al basal. Al expresar el GER por unidad de MLG no hubo variaciones significativas en los valores promedio entre ninguno de los periodos evaluados (Figura 1). Sin embargo, al clasificar a las pacientes por terciles de GER/MLG (Figura 2), se observó que el tercil inferior aumentó

Tabla 2. Cambios en el metabolismo energético en 23 pacientes con seguimiento hasta el primer año postoperatorio

\begin{tabular}{|lccc|}
\hline Parámetro & Mes 0 & Mes 6 & Mes 12 \\
\hline GER (kcal/día) & $1923 \pm 289^{\mathrm{a}}$ & $1631 \pm 256^{\mathrm{b}}$ & $1569 \pm 221^{\mathrm{b}}$ \\
GER/kg (kcal/kg) & $16,9 \pm 22^{\mathrm{a}}$ & $20,2 \pm 2,4^{\mathrm{b}}$ & $21,7 \pm 3,4^{\mathrm{b}}$ \\
GER/kg MLG (kcal/kg MLG) & $32,3 \pm 3,9$ & $34,2 \pm 4,7$ & $32,7 \pm 5,1$ \\
Cuociente respiratorio & $0,75 \pm 0,08$ & $0,69 \pm 0,12$ & $0,71 \pm 0,12$ \\
Oxidación de lípidos (\%) & $73 \pm 17$ & $77 \pm 17$ & $74 \pm 21$ \\
N & 23 & 23 & 23 \\
\hline
\end{tabular}

GER: gasto energético de reposo medido con calorimetría indirecta. GER/kg: gasto energético de reposo por $\mathrm{k}$ de peso corporal total. GER/kg MLG: gasto energético de reposo por k de masa libre de grasa. Supraíndice con letra distinta en la misma fila significa que las diferencias son estadísticamente significativas ( $\mathrm{p} \varangle 0,05)$.

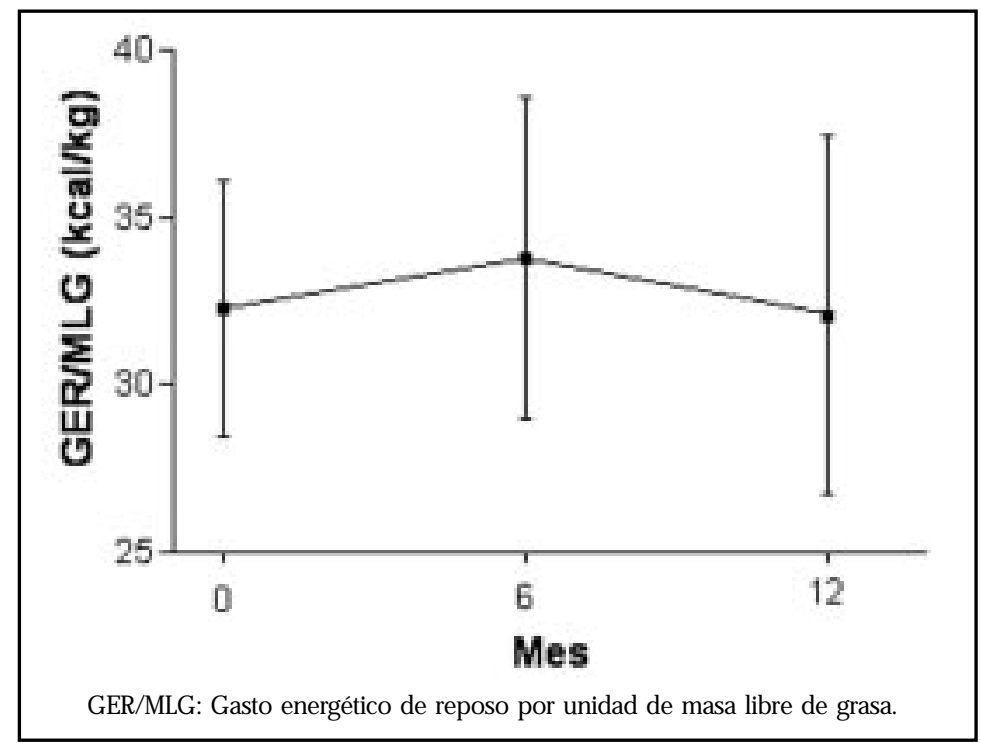

Figura 1. Variación en el gasto energético de reposo por unidad de masa libre de grasa post bypass gástrico (promedio \pm desviación estándar). 


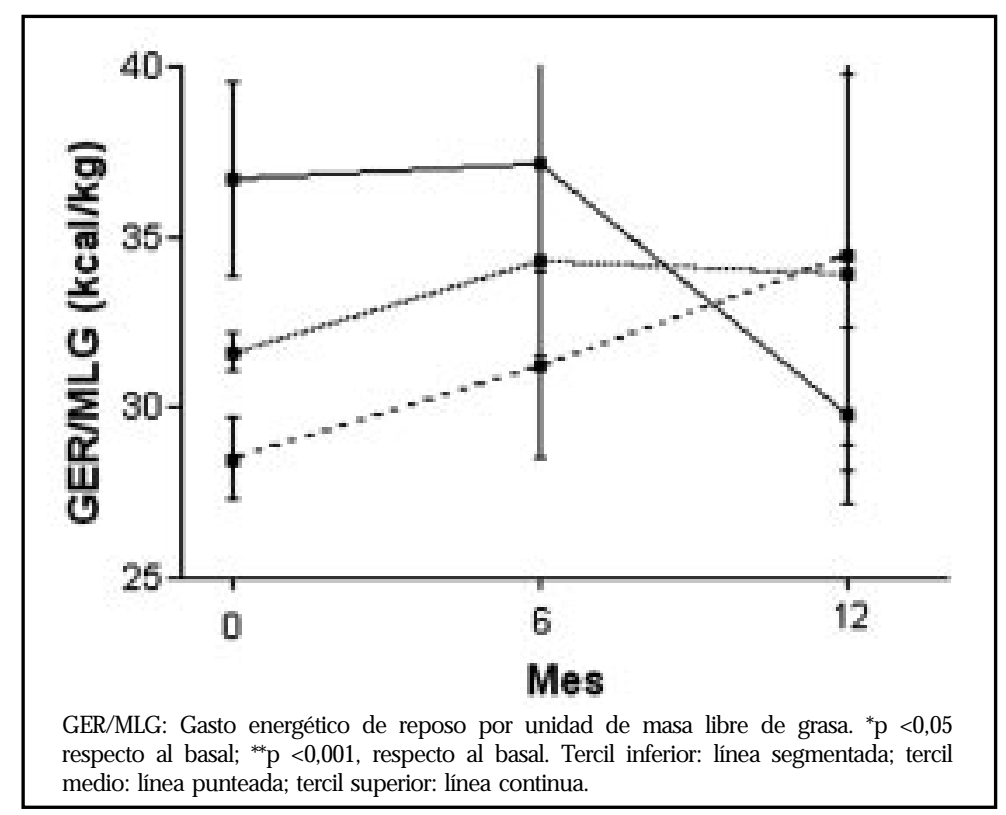

Figura 2. Variación en el gasto energético de reposo por unidad de masa libre de grasa post bypass gástrico según terciles (promedio \pm desviación estándar).

en forma significativa el GER/MLG al mes 6 respecto del basal $(p<0,05)$. El tercil medio aumentó en forma no significativa el GER/MLG durante el postoperatorio, mientras que en el tercil superior el GER/MLG disminuyó en forma significativa al mes 12 ( $p<0,001)$ respecto al preoperatorio.

El cuociente respiratorio y el porcentaje de oxidación de lípidos no variaron en forma significativa.

El GER al mes 0 se correlacionó en forma positiva con el peso $(r=0,596 ; p<0,01)$, la talla ( $r$ $=0,682 ; p<0,001)$, la MLG $(r=0,624 ; p<0,01)$ y la MG en kilos ( $r=0,439 ; p<0,05)$ y se correlacionó en forma negativa con la edad $(r=0,528 ; p$
$<0,05)$. En un análisis de regresión lineal simple, la MLG explica 39\% de la variación del GER inicial ( $\mathrm{r}$ $=0,624 ; r^{2}=0,389$; error estimación: $231 \mathrm{kcal} ; \mathrm{p}$ $<0,01$ ). La MG y la MLG en kilos explican $44 \%$ de la variación del GER inicial $\left(r=0,663 ; r^{2}=0,440\right.$; error estimación: $227 \mathrm{kcal} ; \mathrm{p}<0,01)$. Después de realizar un análisis multivariado la ecuación que mejor predice el GER inicial es: GER (kcal/día) = 121,4 + 30,2 x MLG $(\mathrm{kg})(\mathrm{r}=0,624$; error estimación: $231 \mathrm{kcal}, \mathrm{p}<0,01)$.

$\mathrm{Al}$ mes 6 el GER se correlacionó con el peso, la MLG y la MG en kg, mientras que al mes 12 no se correlacionó con ninguno de estos parámetros en forma significativa. En la Tabla 3 se muestran las

Tabla 3. C oeficientes de correlación del gasto energético de reposo (GER) con parámetros de composición corporal en el periodo preoperatorio y en el mes 6 y 12 postoperatorio

\begin{tabular}{|lllr|}
\hline Parámetro & Mes 0 & Mes 6 & Mes 12 \\
\hline Peso & $0,596^{* *}$ & $0,629^{* *}$ & 0,380 \\
MLG (kg) & $0,624^{* *}$ & $0,476^{*}$ & 0,136 \\
MG (kg) & $0,439^{*}$ & $0,547^{* *}$ & 0,389 \\
\hline
\end{tabular}

GER expresado en kcal/día. MLG: masa libre de grasa; MG: masa grasa; ${ }^{*}<<0,05$; **p $<0,01$. 
correlaciones univariadas del GER medido con las variables de composición corporal, en las distintas etapas de la evolución de las pacientes.

La Tabla 4 muestra la ingesta de macronutrientes en el mes 0, 6 y 12 post BPG. Las correlaciones entre ingesta energética $(\mathrm{kcal} / \mathrm{kg})$ y proteica $(\mathrm{g} / \mathrm{kg})$ y el GER ( $\mathrm{kcal} /$ día y $\mathrm{kcal} / \mathrm{kg} /$ día) no fueron significativas al sexto ni al duodécimo mes postoperatorio. Tampoco hubo correlaciones significativas entre el cambio en la ingesta de estos macronutrientes y el cambio en el GER.

\section{DisCUSIÓN}

El BPG es una técnica eficaz para disminuir el peso de pacientes con obesidad mórbida. En este estudio, las pacientes bajaron $28,4 \%$ del peso inicial al sexto mes y $35 \%$ del peso inicial al año de la operación, cifras similares a las observadas en otros estudios $8,18,24$. La reducción de MLG correspondió a 30\% del peso reducido al año postoperatorio; esta disminución es similar a la observada con tratamientos médicos ${ }^{25,26}$ y quirúrgicos ${ }^{18,24}$ contra la obesidad, aunque mayor que la reportada por otros estudios ${ }^{14}$. La pérdida de MLG podría limitarse con actividad física ${ }^{27}$ y una ingesta adecuada de proteínas ${ }^{18}$.

La disminución significativa del GER de $\sim 300$ $\mathrm{kcal} /$ día al sexto mes y al año postperatorio, podría explicarse fundamentalmente por la reducción en la masa libre de grasa, pero también por los cambios en la ingesta de energía que condicionarían un adaptación metabólica negativa. En nuestro estudio no se observaron diferencias significativas en el GER entre el sexto mes y el año postoperatorio, probablemente por la menor reducción de peso de este periodo. Sin embargo, otros estudios han encontrado que la declinación del GER se prolonga en el tiempo ${ }^{18}$. Esta discordancia podría deberse, entre otros factores, a la edad de las pacientes evaluadas en los distintos estudios: en mujeres entre 51 y 81 años el GER disminuye en forma significativa, no así en menores de 50 años ${ }^{28}$, grupo etario al que pertenecen las pacientes de este estudio.

$\mathrm{Si}$ bien el gasto energético disminuyó en forma significativa, al expresarlo por unidad de masa libre de grasa no se observaron cambios significativos en ninguno de los periodos evaluados, lo cual es concordante con otros estudios ${ }^{18}$. Sin embargo, al analizar según terciles de GER inicial, el grupo con un menor gasto energético aumenta en forma significativa su metabolismo, mientras que el tercil superior lo disminuye en forma significativa durante el postoperatorio. Se podría plantear que los individuos con mayor ingesta de energía en el preoperatorio, como mecanismo compensatorio aumentarían su GER, y la restricción calórica post BPG, que sería proporcionalmente mayor en este grupo de pacientes, produciría una mayor disminución del GER en ellos $^{14,24}$. Sin embargo, no se encontró una correlación significativa entre la disminución de la

Tabla 4. Variación en la ingesta de macronutrientes post bypass gástrico

\begin{tabular}{|lccc|}
\hline Ingesta & Mes 0 & Mes 6 & Mes 12 \\
\hline Calorías (kcal/día) & $1.608 \pm 752^{\mathrm{a}}$ & $815 \pm 207^{\mathrm{b}}$ & $1037 \pm 208^{\mathrm{c}}$ \\
Proteínas (g/día) & $69,1 \pm 27,4^{\mathrm{a}}$ & $44,6 \pm 17,9^{\mathrm{b}}$ & $46,9 \pm 15,3^{\mathrm{b}}$ \\
Hidratos de Carbono (g/día) & $219,3 \pm 111,6^{\mathrm{a}}$ & $101,3 \pm 27,1^{\mathrm{b}}$ & $129,3 \pm 30,1^{\mathrm{c}}$ \\
Lípidos (g/día) & $48,8 \pm 30,0^{\mathrm{a}}$ & $26,0 \pm 8,8^{\mathrm{b}}$ & $36,5 \pm 8,0^{\mathrm{a}}$ \\
Proteínas (\%) & $17,7 \pm 3,6^{\mathrm{a}}$ & $21,5 \pm 5,0^{\mathrm{b}}$ & $18,1 \pm 5,0^{\mathrm{a}}$ \\
Hidratos de carbono (\%) & $54,5 \pm 6,6$ & $50,2 \pm 8,1$ & $50,1 \pm 8,5$ \\
Lípidos (\%) & $26,8 \pm 7,8^{\mathrm{a}}$ & $28,7 \pm 6,4^{\mathrm{a}}$ & $32,0 \pm 6,3^{\mathrm{b}}$ \\
N & 23 & 23 & 23 \\
\hline
\end{tabular}

Supraíndice con letra distinta en la misma fila significa que las diferencias son estadísticamente significativas $(\mathrm{p}<0,05)$. 
ingesta energética y el gasto energético por unidad de masa libre de grasa. El aumento significativo del GER por unidad de peso corporal total al mes 6 y mes 12 podría explicarse por una normalización de la composición corporal de los sujetos en estudio (aumento en la proporción de masa libre de grasa con disminución de la masa grasa).

En conclusión, el bypass gástrico produce cambios significativos en la composición corporal durante el primer año postoperatorio, principalmente por reducción en el compartimiento graso. Durante este período se observa una disminución en el gasto energético de reposo, aunque ésta es significativa sólo en los primeros 6 meses post BPG. A pesar de la significativa reducción de peso, el gasto energético de reposo por unidad de masa libre de grasa no varió en forma significati-

\section{REFERENCIAS}

1. Buchwald H, Avidor Y, Braunwald E, Jensen MD, Pories W, Fahrbach K et al. Bariatric Surgery A Systematic Review and Meta-analysis. JAMA 2004; 292: 1724-37.

2. Carrasco F, Kiahssen J, Papapietro K, Reyes E, Rodríguez L, Csendes A et al. Propuesta y fundamentos para una norma de manejo quirúrgico del paciente obeso. Año 2004. Rev Méd Chile 2005; 133: 699-706.

3. Ocón J, Pérez S, Gimeno S, Benito P, García R. Eficacia y complicaciones de la cirugía bariátrica en el tratamiento de la obesidad mórbida. Nutr Hosp 2005; 20: 409-14.

4. Mizón C, Atalah E. Transición epidemiológica en Chile: lecciones aprendidas del proyecto North Karelia. Rev Chil Nutr 2004; 31: 276-82.

5. MINSAL. I Encuesta de Salud Chile 2003. http:// epi.minsal.cl/epi/html/invest/ENS/InformeFinalENS.pdf

6. Sarwer D, Wadden T, Fabricatore A. Psychosocial and Behavioral Aspects of Bariatric Surgery. Obes Res 2005; 13: 639-48.

7. Colquitt J, Clegg A, Sidhu M, Royle P. Surgery for morbid obesity. Cochrane Database Syst Rev 2003; (2): CD003641.

8. Sjöström L, Lindroos A-K, Peltonen M, Torgerson J, Bouchard C, Carlsson B et al. Lifestyle, Diabetes, va, con lo cual podemos descartar, al menos en nuestro estudio, la aparición de una adaptación metabólica negativa en mujeres sometidas a bypass gástrico en $\mathrm{Y}$ de Roux.

\section{Agradecimientos}

Los autores expresan su agradecimiento a los siguientes profesionales del Hospital Clínico de la Universidad de Chile: Drs. Juan Carlos Díaz, Patricio Burdiles, Fernando Maluenda, Guillermo Watkins y Owen Korn por su participación en la derivación y control de las pacientes. Igualmente, se agradece la colaboración de las Nutricionistas Srtas. Andrea Riffo y Ema Díaz por su participación en la identificación de potenciales candidatas para el estudio y su constante apoyo en los controles posteriores.

and Cardiovascular Risk Factors 10 Years after Bariatric Sungery. N Engl J Med 2004; 351: 268393.

9. Kushner RF, Nobie CA. Long-term outcome of bariatric surgery: an interim analysis. Mayo Clin Proc 2006; 81: S46-51.

10. Csendes A, Burdiles $P$, Papapietro $K$, Díaz JC, Maluenda F, Burgos A et al. Results of gastric bypass plus resection of the distal excluded gastric segment in patients with morbid obesity. J Gastrointest Surg 2005; 9: 121-31.

11. Papapietro K, Díaz E, Csendes A, díaz JC, Braghetto I, BuRdiles P ET AL. Evolución de comorbilidades metabólicas asociadas a obesidad después de cirugía bariátrica. Rev Méd Chile 2005; 133: 511-6.

12. Buchwald H. Consensus Conference Statement Bariatric surgery for morbid obesity: Health implications for patients, health professionals, and third-party payers. J Am Coll Surg 2005; 200: 593604.

13. Csendes A, Burdiles P, Jensen C, Díaz JC, Cortés $C$, Rojas J ET AL. Resultados preliminares de la gastroplastia horizontal con anastomosis en Y de Roux como cirugía bariátrica en pacientes con obesidad severa y mórbida. Rev Méd Chile 1999; 127: 953-60.

14. Das SK, Roberts SB, Mccrory MA, Hsu LK, Shikora SA, KeHAYiAs JJ ET AL. Long-term changes in energy expenditure and body composition after massive 
weight loss induced by gastric bypass surgery. Am J Clin Nutr 2003; 78: 22-30.

15. Astrup A, Gotzsche PC, Van De Werken K, RanNeRIES C, Toubro S, Raben A et al. Meta-analysis of resting metabolic rate in formerly obese subjects. Am J Clin Nutr 1999; 69: 1117-22.

16. CaRRasco F, Rojas P. Metabolismo energético en el obeso mórbido. Rev Hosp Clín Univ Chile 2005; 16: 267-71.

17. Flancbaum L, Choban PS, Bradiey LR, Burge JC. Changes in measured resting energy expenditure after Roux-en-Y gastric bypass for clinically severe obesity. Surgery 1997; 122: 943-9.

18. Carey DG, Plego GJ, Raymond RL, Skau KB. Body composition and metabolic changes following bariatric surgery: effects on fat mass, lean mass and basal metabolic rate. Obes Surg 2006; 16: 469-77.

19. National Institutes of Health Consensus Development Conference Statement. Gastrointestinal surgery for severe obesity. Am J Clin Nutr 1992; 55: $615 S S S$.

20. Johnson RE. Techniques for measuring gas exchange. In: Kinney JM. Assessment of energy metabolism in health and disease. Columbus Ross Laboratories 1980; 32-62.

21. Takala J, Keinanen O, Vaisanen P, Kari A. Measurement of gas exchange in intensive care: laboratory and clinical validation of a new device. Crit Care Med 1989; 17: 1041-7.
22. HaRRIs JA, BENEDICT FG. A biometric study of basal metabolism in man. Washington DC: Carnegie Institute of Washington. Publication № 279, 1919.

23. Carrasco F, Reyes E, NúNezz C, Riedemann K, Rimer O, SáncheZ G ET aL. Gasto energético de reposo medido en obesos y no obesos: comparación con la estimación por fórmulas y ecuaciones propuestas para población chilena. Rev Méd Chile 2002; 130: 51-60.

24. Carrasco F, Papapietro K, Csendes A, Salazar G, Echenique C, Lusboa C et aL. Changes on Resting Energy Expenditure and Body Composition after Weight Loss Following Roux-en-Y Gastric Bypass. Obes Surg 2007; 17: 608-16.

25. Donneluy JE, Pronk NP, Jacobsen DJ, Pronk SJ, JAKICIC JM. Effects of a very-low-calorie diet and physical-training regimens on body composition and resting metabolic rate in obese females. Am J Clin Nutr 1991; 54: 56-61.

26. Berube-Parent S, Prud'homme D, St-Pierre S, Doucet E, TREMBLAY A. Obesity treatment with a progressive clinical tri-therapy combining sibutramine and a supervised diet-exercise intervention. Int J Obes Relat Metab Disord 2001; 25: 1144-53.

27. Chaston TB, Dixon JB, O'BRien PE. Changes in fatfree mass during significant weight loss: a systematic review. Int J Obes 2007; 31: 743-50.

28. Poehlman ET, Goran Mi, Gardner AW, Ades PA, Arciero PJ, KatzMan-Rooks SM et al. Determinants of decline in resting metabolic rate in aging females. Am J Physiol 1993; 264: E450-E455. 